\title{
Louise Hecht (Hg.), Ludwig August Frankl (1810-1894). Eine jüdische Biographie zwischen Okzident und Orient
}

Hélène Leclerc

\section{OpenEdition}

\section{Journals}

Édition électronique

URL : http://journals.openedition.org/austriaca/466

DOI : 10.4000/austriaca.466

ISSN : 2729-0603

Éditeur

Presses universitaires de Rouen et du Havre

\section{Édition imprimée}

Date de publication : 1 décembre 2018

Pagination : 276-279

ISBN : 979-10-240-1354-1

ISSN : 0396-4590

\section{Référence électronique}

Hélène Leclerc, «Louise Hecht (Hg.), Ludwig August Frankl (1810-1894). Eine jüdische Biographie zwischen Okzident und Orient », Austriaca [En ligne], 87 | 2018, mis en ligne le 01 mars 2020, consulté le 28 janvier 2021. URL : http://journals.openedition.org/austriaca/466 ; DOI : https://doi.org/10.4000/ austriaca.466

Ce document a été généré automatiquement le 28 janvier 2021.

Austriaca. Cahiers universitaires d'information sur l'Autriche 


\title{
Louise Hecht (Hg.), Ludwig August Frankl (1810-1894). Eine jüdische Biographie zwischen Okzident und Orient
}

\author{
Hélène Leclerc
}

\section{RÉFÉRENCE}

Louise Hecht (Hg.), Ludwig August Frankl (1810-1894). Eine jüdische Biographie zwischen Okzident und Orient (= Intellektuelles Prag im 19. und 20. Jahrhundert, Band 10), KölnWeimar-Wien, Böhlau, 2016, 430 p., ISBN : 978-3-412-50374-1, $60 €$.

1 Le volume 10 de la belle collection «Intellektuelles Prag im 19. und 20. Jahrhundert " est consacré à l'écrivain, publiciste, voyageur et orientaliste juif originaire de Chrást en Bohême, Ludwig August Frankl. Cet ouvrage, fruit d'un colloque organisé en 2010 à Olomouc (République tchèque) à l'occasion des deux cents ans de la naissance de Frankl, constitue la première étude exhaustive de l'ensemble de la biographie et de l'œuvre de cet acteur important de la vie culturelle et intellectuelle autrichienne, viennoise et juive du XIX siècle. L'ouvrage n'est toutefois pas conçu comme des actes de colloque, mais se présente comme une "biographie polyphonique», ainsi que le définit Louise Hecht dans une remarquable introduction (p.11-45). Outre les quinze contributions organisées en quatre chapitres étudiant les origines de Frankl et de sa famille («Biographische Welt: Tradition und Akkulturation»), son engagement public (« Öffentliche Welt: Literatur und Revolution»), son action au sein de la communauté juive de Vienne ( Jüdische Welt: Philanthropie und Wissenschaft ») et son engagement patriotique (" Patriotische Welt: Denkmäler, Biographien und Nachrufe »), l'ouvrage se compose de précieuses annexes parmi lesquelles une biographie synthétique de Frankl, l'arbre généalogique de sa famille, une bibliographie sélective de son œuvre ainsi 
qu'une riche bibliographie qui inclut notamment une présentation exhaustive des études consacrées jusqu'alors à Frankl.

L'ensemble de l'œuvre de Frankl se trouve tour à tour étudié, qu'il s'agisse de l'œuvre poétique, journalistique, de nouvelles, de son récit de voyage à Jérusalem ou encore de son œuvre biographique et à la mémoire de personnalités illustres, voire de son œuvre de collectionneur. L'œuvre poétique est ainsi abordée par Václav Petrbok («Ludwig August Frankl als tschechischer Dichter? », p. 89-120) et Stefan Schmidl, qui s'intéresse aux nombreuses mises en musique des poèmes de Frankl ( Gedichte von Ludwig August Frankl in ihren Vertonungen. Ein Spektrum musikalischer Übersetzungen », p. 183-194) tout en soulignant la place particulière du poème Die Universität, premier poème imprimé sans censure au moment de la révolution de 1848 (p. 183) et devenu grâce à ses différentes versions musicales une sorte de "Marseillaise autrichienne " (p. 184). L'œuvre journalistique intéressent Barbara Boisits, qui étudie la revue Sonntagsblätter - dont Frankl fut le rédacteur de 1842 à 1848 - sous l'angle original de sa contribution à l'élaboration d'une critique musicale viennoise ( Die Bedeutung der Sonntagsblätter Ludwig August Frankls für die Wiener Musikkritik», p. 157-182), ainsi que Gertraud Marinelli-König ( «udwig August Frankl und die Wiener Unterhaltungsblätter im Vormärz », p. 207-220), qui présente Frankl comme «l'une des personnalités du monde des médias les plus influentes » de son époque (p. 207), ou encore Ernst Wangermann, qui examine le rôle de Frankl durant la révolution de 1848 et se concentre à cette fin " essentiellement sur la revue hebdomadaire Sonntagsblätter, à laquelle Frankl conféra un caractère presque exclusivement politique à partir de mars 1848, et au Wiener Abendzeitung qu'il édita à partir de fin mars 1848 comme supplément quotidien des Sonntagsblätter » (p. 195). Carsten L. Wilke propose quant à lui une étude tout à fait passionnante de la contribution jugée primordiale de Frankl, via la nouvelle Diego de Aguilar, à la création d'un mythe marrane («Ludwig August Frankl als historischer Mythograph der Marranen », p. 219-240). L'œuvre de Frankl voyageur et orientaliste est l'objet de deux articles, celui de Marie Krappmann («Ludwig August Frankls „Nach Jerusalem!“. Über die Logik der Widersprüche und das Zerbröckeln der Metaphern », p. 241-256) et celui de Yochai Ben-Ghedalia (" „My Heart is in the East“: Ludwig August Frankl's Mission to the Orient in the Footsteps of Yehuda Halevy ", p. 257-273). Herlinde Aichner ("Ludwig August Frankl - Politiker der Erinnerung », p. 275-290) s'intéresse pour sa part à la mise en œuvre d'une "politique de la mémoire» par Frankl, rédacteur de nombreuses biographies, édificateur de monuments en hommage à ses contemporains ou illustres prédécesseurs et insatiable collecteur d'objets et de documents (Louise Hecht parle de Sammelleidenschaft, p. 297), qui font aujourd'hui la richesse du Musée juif de Vienne en particulier, comme l'indique Gabriele Kohlbauer-Fritz dans son étude «Ludwig August Frankl und das Jüdische Museum» (p. 323). Reprenant la thèse de la material culture selon laquelle les objets qui nous entourent livrent eux aussi un portrait de leur propriétaire (p. 295), Louise Hecht étudie le monde des objets de Frankl et son «musée » privé qu'était devenu son domicile.

3 Cette appétence pour la construction de monuments à la gloire de grands hommes ne fut pas sans susciter d'âpres polémiques, en particulier avec Ferdinand Kürnberger, qui soupçonnait Frankl de tenter de se mettre lui-même en avant par le biais de ces monuments (p. 285). Hubert Lengauer étudie de manière approfondie la relation entre les deux hommes, qui furent d'abord amis; l'évolution de leur amitié et collaboration 
étroite avant 1848 en vive hostilité de la part de Kürnberger illustre aussi la montée de l'antisémitisme en Autriche dans la seconde moitié du XIX siècle (Hubert Lengauer, « Konkurrenz und Kompensation. Ludwig August Frankl und Ferdinand Kürnberger », p.137-156). Un dernier aspect de l'œuvre de Frankl se trouve également remarquablement exploité dans cet ouvrage. Il s'agit de la correspondance foisonnante de l'écrivain, dont tire profit notamment Dieter J. Hecht pour mettre en lumière avec minutie la biographie de Frankl à travers ses relations féminines, qui jouèrent un rôle important (« „Wie glücklich ist sicher die gute Paula..." Frauen im Leben von Ludwig August Frankl », p. 47-76).

4 L'ensemble de ces contributions met en évidence deux caractéristiques de l'œuvre et du parcours de Frankl. La première est sa position d'entre-deux, d'emblée soulignée par le sous-titre du volume, entre-deux qui confère un statut de passeur, voire de médiateur, mais qui dépasse largement le cadre des relations entre monde occidental et Orient, entre christianisme et judaïsme. Václav Petrbok («Ludwig August Frankl als tschechischer Dichter?», p. 89-120) souligne ainsi sa situation entre Tchèques et Allemands, entre identité allemande et identité tchèque, langue allemande et langue tchèque, littérature allemande et littérature tchèque. Jörg Krappmann ( Der "Mitgenannte“. Ludwig August Frankl und die Literaturgeschichte », p. 121-136) évoque quant à lui une œuvre littéraire «zwischen biedermeierlicher Weltschmerzdichtung und revolutionärem Vormärz, und damit zwischen Skylla und Charybdis der Literaturgeschichtsschreibung des 19. Jahrhunderts » (p. 135). Selon Jörg Krappmann, cette situation d'entre-deux dans l'histoire littéraire expliquerait le "processus de marginalisation » (p. 122) dont Frankl a finalement été victime, pour lequel Krappmann préfère forger le terme de Mitgenannter, plutôt que de reprendre des concepts déjà existants tels que Vernachlässigter, Vergessener ou Verschollener qui ne correspondent pas vraiment à la situation de Frankl (p.122). Nous abordons ainsi la seconde caractéristique de l'œuvre de Frankl qui ressort particulièrement de cette «biographie collective» (Hecht, p. 45), à savoir sa place en marge du canon de la littérature et de l'histoire autrichienne en dépit d'une carrière tout à fait remarquable dans le monde des lettres et dans la vie culturelle autrichienne.

5 À travers la biographie de Frankl, c'est l'histoire de l'Autriche du XIX siècle et du judaïsme en Autriche qui se trouve éclairée. 